\title{
Current trends in molecular magnetic resonance imaging of the extracellular matrix in atherosclerosis
}

\author{
Mangarova D ${ }^{1,2, *}$, Brangsch J ${ }^{1,3}$, Möckel J ${ }^{1}$, Kader A ${ }^{1,4}$, Kaufmann JO ${ }^{1,5,6}$, Ludwig A ${ }^{1}$, Taupitz M $^{1}$, Hamm B ${ }^{1}$ and Makowski MR ${ }^{1,7}$ \\ ${ }^{1}$ Charité - Universitätsmedizin Berlin, Corporate Member of Freie Universität Berlin, Humboldt-Universität zu Berlin, and Berlin Institute of Health, Charitéplatz \\ 1, 10117 Berlin, Germany \\ ${ }^{2}$ Department of Veterinary Medicine, Institute of Veterinary Pathology, Freie Universität Berlin, Robert-von-Ostertag-Str. 15, Building 12, 14163 Berlin, Germany \\ ${ }^{3}$ Department of Veterinary Medicine, Institute of Animal Welfare, Animal Behavior and Laboratory Animal Science, Freie Universität Berlin, Königsweg 67, \\ Building 21, 14163 Berlin, Germany \\ ${ }^{4}$ Department of Biology, Chemistry and Pharmacy, Institute of Biology, Freie Universität Berlin, Königin-Luise-Str. 1-3, 14195 Berlin, Germany \\ ${ }^{5}$ Federal Institute for Materials Research and Testing (BAM), Division 1.5 Protein Analysis, Richard-Willstätter-Str. 11, 12489 Berlin, Germany \\ ${ }^{6}$ Humboldt-Universität zu Berlin, Department of Chemistry, Brook-Taylor-Str. 2, 12489 Berlin, Germany \\ ${ }^{7}$ Technical University of Munich, Department of Diagnostic and Interventional Radiology, Ismaninger Str.22, 81675 Munich, Germany
}

\begin{abstract}
Atherosclerosis is the underlying pathology of the majority of cardiovascular events in the Western world. It is well established that both the cellular and extracellular components of the arterial wall undergo morphological changes during plaque development. The extracellular matrix (ECM), a network of various macromolecules such as collagen, proteoglycans, elastin and fibrin, not only provides structural support for the vessel wall, but plays also a key role for biological signaling and cell interactions. Current standard clinical imaging modalities are limited to detecting anatomical changes in atherosclerosis. In recent years, molecular magnetic resonance imaging (MRI) has emerged as a promising in vivo alternative. Molecular MRI enables the visualization of biological changes in the formation and progression of plaques and the development of novel ECM-targeting MRI contrast agents has been on the rise. This review presents a summary of the recent advancements in the field of molecular probes for ECM imaging in the context of atherosclerosis.
\end{abstract}

\begin{abstract}
Abbreviations: Apo-E -/-: Apolipoprotein e-deficient mice; HDL: High-densitiy lipoprotein; ESMA: Elastin-specific magnetic resonance imaging agent; USPIO: Ultrasmall superparagnetic iron-oxide; TESMA: Tropoelastin-specific magnetic resonance imaging agent; GAG: Glycosaminoglycans; VSOP: Very small superparamagnetic iron oxide particles; LDLR -/- : Low-density lipoprotein receptor-deficient mice.
\end{abstract}

\section{Introduction}

Currently, cardiovascular diseases are accountable for almost one-third of sudden and premature death cases worldwide [1]. Atherosclerosis is responsible for the majority of high mortality cardiovascular and cerebrovascular clinical manifestations such as stroke and acute myocardial infarction. It is defined by the formation of plaque in arterial vessel walls as a result of multifactorial alterations of both the cellular and extracellular components of the artery. The extracellular matrix (ECM) is an ubiquitary element of both healthy and diseased tissues. This is equally valid for atherosclerotic plaques, whose mass is to a considerable extent formed by ECM components such as collagen, elastin, fibrin, glycosaminoglycans (GAGs) and proteoglycans. The progression of atherosclerosis is regulated by the fine balance of disintegration and synthesis of these biomolecules.

The current clinical imaging techniques, namely coronary angiography and contrast-enhanced computer tomography concentrate on relative plaque size to calculate the degree of luminal stenosis. Even though these imaging modalities are the current standard diagnostic reference, they only serve as an indirect marker and underestimate plaque burden, considering that compensatory vessel enlargement can sustain a plaque mass increase with no luminal narrowing [2].

Magnetic resonance imaging (MRI) has emerged as a radiationfree technique to directly and effectively image plaque depositions. Molecular MRI expands the information about plaque anatomy by additional evaluation of plaque morphology and physiology, both crucial for profiling cardiovascular event risk [3].

A growing body of literature has evaluated the development of novel cardiovascular molecular probes (Figure 1). The goal of this literature review is to discuss the recent advances in the development of ECM-targeting contrast agents and their application in the context of atherosclerosis.

${ }^{\star}$ Correspondence to: Dilyana B. Mangarova, DVM, Department of Radiology, Charité - Universitätsmedizin Berlin, Charitéplatz 1, 10117 Berlin, Germany, E-mail: dilyana.mangarova@charite.de

Key words: atherosclerosis, collagen, elastin, fibrin, molecular imaging, proteoglycan

Received: May 02, 2020; Accepted: May 22, 2020; Published: May 25, 2020 


\begin{tabular}{|c|c|c|c|}
\hline Molecular target & MRI agent & Animal model & Investigators \\
\hline \multirow[t]{3}{*}{ Collagen } & EP-3533 & Infareted C57BL/6 mouse & Caravan et al. (2007) \\
\hline & HDL- EP-3533 & Reversa mouse & Chen et al. (2013) \\
\hline & Platelet membrane-coated nanoparticles & Apo-E -/- mouse & Wei et al. (2018) \\
\hline \multirow[t]{6}{*}{ Elastin } & ESMA & Apo-E-/-mouse & Makowski et al. (2011) \\
\hline & & Landrace pig & von Bary et al. (2011) \\
\hline & & New Zealand white rabbit & Phinikaridou et al. (2014) \\
\hline & ESMA + USPIO & Apo-E -/-mouse & Reimann et al. (2019) \\
\hline & TESMA & Apo-E - /-mouse & Phinikaridou et al. (2018) \\
\hline & & New Zealand white rabbit & Phinikaridou et al. (2018) \\
\hline \multirow[t]{3}{*}{ Fibrin } & EP-1873 & New Zealand white rabbit & Botnar et al. (2004) \\
\hline & EP-2104R & Coronary thrombosis domestic pig & Botnar et al. (2004) \\
\hline & EP-1242 & Guinea pig & Sirol et al. (2005) \\
\hline \multirow[t]{2}{*}{ GAG } & VSOP & Watanabe heritable hyperlipidemic rabbit & Wagner et al. (2013) \\
\hline & & LDLR $-/-$ mouse & Poller et al. (2016) \\
\hline
\end{tabular}

Figure 1. Classification of the novel ECM targeting probes discussed in this review categorized in following groups: (1) collagen, (2) elastin, (3) fibrin and (4) GAG

\section{Molecular magnetic resonance imaging of the extracellular matrix}

\section{Collagen-targeting probes}

The collagen protein family consists of around 20 genetically different subtypes [4], with subtypes I, III, IV, V, VI and VIII involved in the composition of arterial vessels [5]. While subtype $I$ is the predominant form in healthy tissues [6], types I, III, IV and V are highly expressed in ECM of atherosclerotic plaques, with type IV found in the fibrous caps of plaques and type $\mathrm{V}$ especially common in advanced atheromas [7]. These characteristics make collagen an ideal surrogate marker for ECM remodeling.

The overall amount and length of collagen proteins in plaques is critical for risk assessment. Following atherosclerosis initiation, the expression of collagen increases greatly $[8,9]$ and can contribute to up to $60 \%$ of proteins in the ECM of atherosclerotic plaques [10], being responsible for maintaining the plaques integrity and reducing the danger of rupture. Activated matrix metalloproteinases (MMP1, MMP 8, MMP13) [11,12], secreted by macrophages and smooth muscle cells (SMC), fragment long chain collagen chains into short peptides [13]. This process is especially prominent in the fibrous cap region of the plaque so that plaques characterized by highly fragmented collagen fibers and decreased collagen density are prone to rupture $[14,15]$.

EP-3533 - a type I collagen-targeting probe was presented by Caravan et al. in 2007 [16]. This gadolinium-based molecular agent has so far successfully been tested for the specific detection of earlystage fibrosis across various small animal disease models, including myocardial infarction [16], liver fibrosis [17,18] and pulmonary fibrosis [19]. By conjugating EP-3533 with high density lipoprotein (HDL) nanoparticles, it was not only possible to detect atherosclerotic plaques, but also to measure their regression in a mouse plaque regression model [20].

Another approach was recently introduced by Wei et al. [21]. In this study, gadolinium labeled nanoparticles containing platelet membrane around a synthetic nanoparticle core were used in a murine Apo-E -/- model. By mimicking the role of platelets in the pathology of atherosclerosis, these particles interact with collagen, foam cells and activated endothelium at the plaque site. In vitro assay confirmed that this novel agent binds to type IV collagen specifically.

\section{Elastin-targeting probes}

One of the major proteins found in the ECM, making up to half of an artery's dry weight [22] and responsible for the tensile strength and integrity of the physiologically intact arterial wall is elastin [23]. Tropoelastin, elastin's soluble precursor, is mainly expressed by SMC [24] and subsequently cross-linked and merged into circular elastin lamellae in the vessel wall. In the development of atherosclerosis increased elastogenesis, triggered by various biological signals [25] and conversely increased elastolysis, caused by MMPs [26], leading to fragmentation and degradation, result in disrupted composition and appearance of the ECM in plaques.

The increased relative amount of elastin in the plaque matrix represents an excellent molecular target. Recent research efforts led to the development of an elastin-targeting probe. So far, the specificity of this novel contrast agent to elastin was successfully tested both ex vivo and in vivo in small and large animal models [27-30].

The latest advancement in this field is the development of a simultaneous dual-probe molecular MRI protocol in a single imaging session, combining the assessment of plaque burden with inflammatory activity in an atherosclerotic Apo-E -/- mouse model [31]. While the accumulation of the iron-oxide-based contrast agent was most prominent in the early stage of plaque development following two months of high-fat diet (HFD), the elastin-specific probe showed the highest accumulation in advanced plaques after four months of HFD. Neither of both probes in this study affected the visualization of the other one. Combining different biomarkers related to atherosclerosis could enable better risk assessment of patients in the future.

Another approach to target dysfunctional elastogenesis and elastolysis was developed by Phinikaridou et al. [32]. By targeting 
tropoelastin with a gadolinium-labeled tropoelastin-specific magnetic resonance contrast agent in two animal models, namely Apo-E -/- mice and New Zealand white rabbits, a novel biomarker for plaque progression and instability was obtained. After delayedenhancement MRI (DE-MRI) of the Apo-E -/- control group, vessel wall enhancement was visible with gadolinium-ESMA (Gd-ESMA), while no enhancement was shown after Gd-TESMA since tropoelastin is not found in healthy arteries. In two mice in the experimental group, vessel wall enhancement after DE-MRI was visible after administration of both Gd-ESMA and Gd-TESMA, whereas Gd-ESMA contributed to a stronger enhancement than Gd-TESMA. Since the atherosclerotic vessel wall contains both cross-linked elastin and tropoelastin and GdESMA binds to both, Gd-ESMA contributed to a stronger enhancement than Gd-TESMA.

\section{Fibrin-targeting probes}

Imaging fibrin in the context of atheromas presents as more challenging than imaging elastin and collagen since it is less abundant than the aforementioned. However, fibrin is not a natural component of the arterial wall, establishing fibrin-targeting probes as a promising and highly specific surrogate marker. Not only is fibrin a key player in the coagulation cascade after plaque rupture, but it is also highly relevant during plaque progression [33]. The increased endothelial permeability leads to fibrin influx [34], adding to the absolute plaque ECM mass [35] and serving as an adhesion point for proinflammatory cells, platelets and SMC [36,37]. It has been shown that the relative amount of fibrin in advanced plaques increases and is especially high in the necrotic core [33].

Botnar et al. developed the fibrin-targeted gadolinium-based peptide probe EP-1873 with high relaxivity and stability [38], called EP1873 that was subsequently tested in an atherosclerotic New Zealand white rabbit model. Plaque rupture was induced, and the rupture site was visible in MRI with a $100 \%$ overlap to the ex vivo histological findings [38]. EP-1873s stability and binding specificity to fibrin was further optimized, leading to the development of EP-2104R [39]. So far, this probe has been applied in large and small animal models of arterial, venous, coronary and atrial thrombosis as well as pulmonary and cerebral embolism [40-43]. More important, it was also possible to differentiate between thrombi that tend to thrombolysis and stable thrombi based on their fibrin content [44]. Further experimental investigations need to be performed to establish whether EP-2104R could also be useful as a pre-rupture molecular agent in the earlier stages of plaque development.

EP-1242 is another fibrin-targeted small peptide derivative that was successfully tested in an acute carotid thrombosis guinea pig model [45]. After rapidly binding to the fibrin-rich surface area of the clot, the contrast agent disseminates in the thrombus within 30 minutes after intravenous injection. The invasive nature of this model restrict it to the detection of acute non-occlusive thrombi only, excluding the detection of non-occlusive and subacute thrombi.

\section{Glycosaminoglycan-targeting probes}

GAGs are fundamental building blocks for proteoglycans, playing an important role in the development and progression of atheroma by interactions with LDL, collagen, cytokines and platelets [46,47]. Similar to collagen fibers, the degradation of GAG chains contributes to plaque instability $[48,49]$. The most common proteoglycans found in healthy arterial walls are biglycan, syndecan, perlecan, decorin and versican, with the last-mentioned also highly expressed in human atherosclerotic lesions. However, in murine models, biglycan and perlecan, but not versican accumulate in atherosclerotic plaques [50].

Several in vitro and in vivo studies investigated the interactions of very small superparamagnetic iron oxide particles (VSOP) with atherosclerosis-associated cell types and extracellular structures [5153]. The results suggested that the interaction of pathologically increased GAGs influences both the uptake of VSOP into atherosclerotic lesions and the accumulation within the diseased tissue, thereby enabling non-invasive MRI assessment of plaque inflammation and increased endothelial permeability $[52,53]$.

\section{Conclusion and perspectives}

In conclusion, this minireview represents an overview of the current developments in ECM-targeting MRI probes. Molecular MR imaging is an emerging and promising non-invasive modality for the characterization of pathobiological mechanisms in the progression of atherosclerosis. ECM, being a substantial and ubiquitous component of both healthy and diseased tissues plays a key role as a molecular target. Translational research allows the application and in vivo visualization of these contrast agents, verifying their specificity and efficacy in animal models and bringing the development of novel molecular MR imaging probes one step closer to a clinical setting.

\section{Conflicts of interest}

The author declares no competing interests.

\section{Sources of funding}

This study was funded by the Deutsche Forschungsgemeinschaft (DFG, German Research Foundation) - Projektnummer 372486779 SFB 1340; MA 5943/3-1/4-1/9-1.

\section{Author contributions}

D.B.M. performed the literature review and wrote the manuscript. M.R.M. designed and supervised the study. J.B., J.M., A.K., J.O.K, M.T, B.H and A.L. reviewed, edited and commented on the manuscript.

\section{References}

1. Sidney S (2013) The "heart disease and stroke statistics--2013 update" and the need for a national cardiovascular surveillance system. Circulation 127: 21-23.

2. Glagov SWE, Zarins CK, Stankunavicius R, Kolettis GJ (1987) Compensatory enlargement of human atherosclerotic coronary arteries. N Engl J Med 316: 1371-1375. [Crossref]

3. Marnane M (2014) Plaque inflammation and unstable morphology are associated with early stroke recurrence in symptomatic carotid stenosis. Stroke 45: 801-806.

4. Prockop DJ (1995) Collagens: molecular biology, diseases, and potentials for therapy Annu Rev Biochem 64: 403-434.

5. Mayne R (1986) Collagenous proteins of blood vessels. Arteriosclerosis 6: 585-593

6. Murata K (1986) Collagen types in various layers of the human aorta and their changes with the atherosclerotic process. 1986.

7. Ooshima YM (1990) Collagen metabolism in atherogenesis. Ann N Y Acad Sci 598: 582-584. [Crossref]

8. Yasuda O (2000) Differential expression of the alpha 1 type VIII collagen gene by smooth muscle cells from atherosclerotic plaques of apolipoprotein-E-deficient mice. J Vasc Res 37: 158-169.

9. Plenz G (1999) Expression of type VIII collagen after cholesterol diet and injury in the rabbit model of atherosclerosis. Arterioscler. Thromb Vasc Biol 19: 1201-1209.

10. Smith EB (1965) The influence of age and atherosclerosis on the chemistry of aortic intima. 2. Collagen and mucopolysaccharides. J Atheroscler Res 5: 241-248. 
11. Visse R (2003) Matrix metalloproteinases and tissue inhibitors of metalloproteinases: structure, function, and biochemistry. Circ Res 92: 827-839.

12. Brinckerhoff CE (2002) Matrix metalloproteinases: a tail of a frog that became a prince. Nat Rev Mol Cell Biol 3: 207-214. [Crossref]

13. Miller EJ (1976) Cleavage of type II and III collagens with mammalian collagenase: site of cleavage and primary structure at the NH2-terminal portion of the smaller fragment released from both collagens. Biochemistry 15: 787-792. [Crossref]

14. Lee RT (1996) Circumferential stress and matrix metalloproteinase 1 in human coronary atherosclerosis, implications for plaque rupture. Arterioscler Thromb Vasc Biol 16: 1070-1073. [Crossref]

15. Fujii K (2003) Intravascular ultrasound assessment of ulcerated ruptured plaques: a comparison of culprit and nonculprit lesions of patients with acute coronary syndromes and lesions in patients without acute coronary syndromes. Circulation 108: 2473-2478. [Crossref]

16. Caravan P (2007) Collagen-targeted MRI contrast agent for molecular imaging of fibrosis. Angew Chem Int Ed Engl 46: 8171-8173.

17. Polasek M (2011) Molecular MR Imaging of Liver Fibrosis with a Collagen-Targeting Gadolinium-Based Contrast Agent. Proc Intl Soc Mag Reson Med 19.

18. Zhu B (2017) Combined magnetic resonance elastography and collagen molecular magnetic resonance imaging accurately stage liver fibrosis in a rat model. Hepatology 65: 1015-1025.

19. Caravan P (2013) Molecular magnetic resonance imaging of pulmonary fibrosis in mice. Am J Respir Cell Mol Biol 49: 1120-1126.

20. Chen W (2013) Collagen-Specific Peptide Conjugated HDL Nanoparticles as MRI Contrast Agent to Evaluate Compositional Changes in Atherosclerotic Plaque Regression. JACC: Cardiovascular Imaging 6: 373-384.

21. Wei X (2018) Nanoparticle Functionalization with Platelet Membrane Enables Multifactored Biological Targeting and Detection of Atherosclerosis. ACS Nano 12: 109-116.

22. Parks WC (1993) The extracellular matrix. Adv Mol Cell Biol 6: 133-182.

23. Brasselet C (2005) Collagen and elastin cross-linking: a mechanism of constrictive remodeling after arterial injury. Am J Physiol Heart Circ Physiol 289: H2228-H2233.

24. Krettek A (2003) Elastogenesis in human arterial disease: a role for macrophages in disordered elastin synthesis. Arterioscler Thromb Vasc Biol 23: 582-587.

25. Isik C (1994) Elastin expression in a model of acute arterial graft rejection. Transplantation.

26. Matrisian LM (1992) The matrix-degrading metalloproteinases. Bioessays 14: 455-463.

27. Makowski MR (2011) Assessment of atherosclerotic plaque burden with an elastinspecific magnetic resonance contrast agent. Nat Med 17: 383-388. [Crossref]

28. von Bary C (2011) MRI of coronary wall remodeling in a swine model of coronary injury using an elastin-binding contrast agent. Circ Cardiovasc Imaging 4: 147-155.

29. Onthank D (2007) A novel low molecular weight magnetic resonance contrast agent selective for arterial wall imaging. Circulation 116 ( II 411-II 412).

30. Phinikaridou MEA (2014) Vascular remodeling and plaque vulnerability in a rabbit model of atherosclerosis: comparison of delayed-enhancement MR imaging with an elastin-specific contrast agent and unenhanced black-blood MR imaging. Radiology 271: 390-399.

31. Reimann C (2019) Dual-probe molecular MRI for the in vivo characterization of atherosclerosis in a mouse model: Simultaneous assessment of plaque inflammation and extracellular-matrix remodeling. Sci Rep 9: 13827.

32. Phinikaridou A (2018) Tropoelastin: A novel marker for plaque progression and instability. Circ Cardiovasc Imaging 11.
33. Tavora $F(2010)$ Immunolocalisation of fibrin in coronary atherosclerosis: implications for necrotic core development. Pathology 42: 15-22.

34. Valenzuela R (1992) Immunoelectrophoretic and immunohistochemical characterizations of fibrinogen derivatives in atherosclerotic aortic intimas and vascular prosthesis pseudo-intimas. Am J Pathol 141: 861-880.

35. Falk E (1995) Role of thrombosis in atherosclerosis and its complications. Am J Cardiol 75 (3B-11B).

36. Katagiri Y (1995) Involvement of alpha $v$ beta 3 integrin inmediating fibrin gel retraction. J Biol Chem 270: 1785-1790.

37. Naito M (1996) Migration of cultured vascular smooth muscle cells into noncrosslinked fibrin gels. Thromb Res 84: 129-136.

38. Botnar RM (2004) In Vivo Molecular Imaging of Acute and Subacute Thrombosis Using a Fibrin-Binding Magnetic Resonance Imaging Contrast Agent. Circulation 109: 2023-2029.

39. Overoye-Chan K (2008) EP-2104R: A fibrin-specific gadolinium-based MRI contrast agent for detection of thrombus. Journal of the American Chemical Society 130: 60256039.

40. Botnar RM (2004) In vivo magnetic resonance imaging of coronary thrombosis using a fibrin-binding molecular magnetic resonance contrast agent. Circulation 110: 14631466.

41. Stracke CP (2007) Molecular MRI of cerebral venous sinus thrombosis using a new fibrin-specific MR contrast agent. Stroke 38: 1476-1481.

42. Spuentrup E (2005) Molecular magnetic resonance imaging of atrial clots in a swine model. Circulation 112: 396-399.

43. Spuentrup E (2005) Molecular magnetic resonance imaging of coronary thrombosis and pulmonary emboli with a novel fibrin-targeted contrast agent. Circulation 111: 1377-1382. [Crossref]

44. Andia ME (2014) Fibrin-targeted magnetic resonance imaging allows in vivo quantification of thrombus fibrin content and identifies thrombi amenable for thrombolysis. Arterioscler Thromb Vasc Biol 34: 1193-1198.

45. Sirol M (2005) Fibrin-targeted contrast agent for improvement of in vivo acute thrombus detection with magnetic resonance imaging. Atherosclerosis 182: 79-85.

46. Wagner WD (1985) Proteoglycan structure and function as related to atherosclerosis Ann NY Acad Sci 454: 52-68.

47. Berenson GS (1984) Recent advances inmolecular pathology. Carbohydrate-protein macromolecules and arterial wall integrity - a role in atherogenesis. Exp Mol Pathol 41: 267-287. [Crossref]

48. Kolodgie FD (2002) Differential accumulation of proteoglycans and hyaluronan in culprit lesions: insights into plaque erosion. Arterioscler Thromb Vasc Biol 22: 16421648. [Crossref]

49. Kruse R (1996) Cholesterol-dependent changes of glycosaminoglycan pattern in human aorta. Basic Res Cardiol 91: 344-352.

50. Kunjathoor VV (2002) Accumulation of biglycan and perlecan, but not versican, in lesions of murine models of atherosclerosis. Arterioscler Thromb Vasc Biol 22: 462 468. [Crossref]

51. Ludwig A (2013) Rapid binding of electrostatically stabilized iron oxide nanoparticles to THP-1 monocytic cells via interaction with glycosaminoglycans. Basic Res Cardiol 108: 328 .

52. Wagner S (2013) Contrast-enhanced MR imaging of atherosclerosis using citratecoated superparamagnetic iron oxide nanoparticles: calcifying microvesicles as imaging target for plaque characterization. Int J Nanomedicine 8: 767-779. [Crossref]

53. Poller WC (2016) Uptake of citrate-coated iron oxide nanoparticles into atherosclerotic lesions in mice occurs via accelerated transcytosis through plaque endothelial cells. Nano Research 9: 3437-3452.

Copyright: (C2020 Mangarova D. This is an open-access article distributed under the terms of the Creative Commons Attribution License, which permits unrestricted use, distribution, and reproduction in any medium, provided the original author and source are credited. 